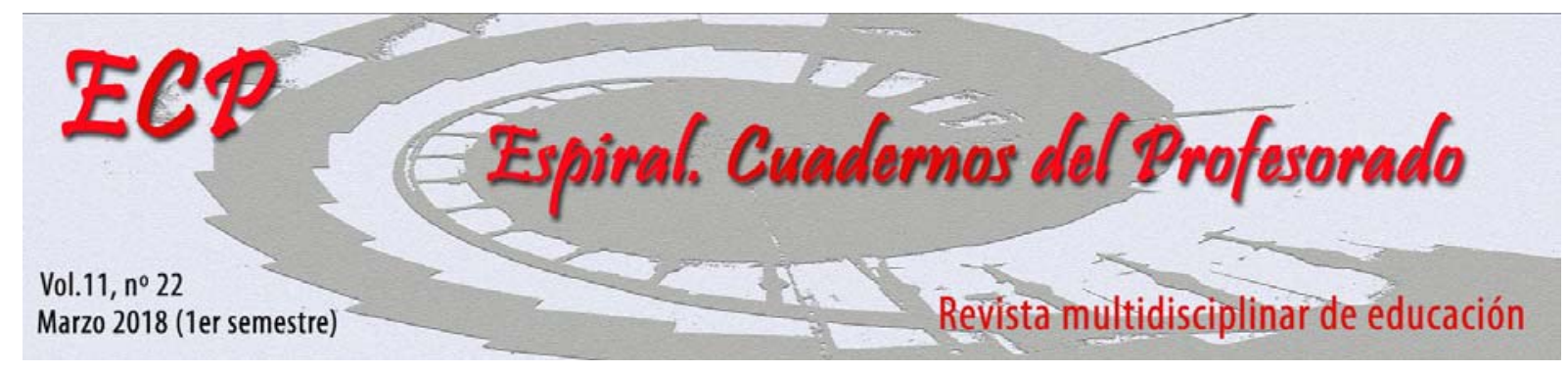

\title{
Aprendizajes Significativos mediante la Gamificación a partir del Juego de Rol: "Las Aldeas de la Historia"
}

\author{
Significant Learning through Gamification, using the Role Game: "The Villages of \\ History"
}

\author{
Jesús Fernández Gavira ${ }^{(1)}$, Ezequiel Prieto Gallego ${ }^{(1)}$, \\ Virginia Alcaraz-Rodríguez ${ }^{(1,2)}$, Antonio J. Sánchez-Oliver ${ }^{(1,2)}$, \\ y Moisés Grimaldi-Puyana ${ }^{(1)}$ \\ (1) Universidad de Sevilla, España \\ (2) Universidad Pablo de Olavide, Sevilla, España
}

\begin{abstract}
RESUMEN: Este trabajo es una propuesta didáctica diseñada desde la perspectiva metodológica de la pedagogía de la aventura, la cual tiene como objetivo educar en y a través de las actividades en la naturaleza, para llevarla a cabo en el contexto escolar y desarrollarla en el primer o segundo ciclo de primaria de Educación Física, siempre teniendo como enfoque el aprendizaje experiencial. Para ello nos hemos centrado en las Habilidades Motrices Básicas (HMB) como contenido principal, las cuales se enseñarán de manera lúdica a través del juego de rol como proyecto interdisciplinar. Las actividades que aquí se proponen, se dividen en dos sesiones. Una primera, en la que se enseñará a los alumnos algunas herramientas necesarias para poder llevar a cabo la segunda sesión, que será el gran juego de rol, el cual se realizará fuera del centro educativo de manera coordinada con otras áreas, que aprovecharán el elemento lúdico del juego para impartir contenidos de las mismas. De manera específica desde el departamento de Educación Física se aprenderán las HMB
\end{abstract}

Palabras clave: Pedagogía de la Aventura; Juego de rol; Habilidades Motrices Básicas; Orientación; Gamificación.

\begin{abstract}
This Work is a didactic proposal designed to carry it out in the school context, and develop it in the first or second cycle of elementary education in Physical Education. For this we have focused on the Basic Motor Skills (HMB) as the main content, which will be taught in a playful way through role play. The activities proposed here are divided into two sessions, a first one, where students will work on some necessary tools to be able to perform the second session, which will be the Great Role Play, which will be taught outside the school in a coordinated way with other areas, which will take advantage of the playful element of the game to teach contents of the same and from the Department of Physical Education in turn to teach HMB.
\end{abstract}

Key words: Pedagogy of Adventure; Role playing game; Basic Motor Skills; Orientation; Gamification.

Fernández Gavira, J., Prieto Gallego, E., Alcaraz-Rodríguez, V., Sánchez-Oliver, A. J., y GrimaldiPuyanal, M. (2018). Aprendizajes significativos mediante la Gamificación a partir del Juego de Rol: "Las Aldeas de la Historia". Espiral. Cuadernos del Profesorado, 11(22), 69-78.

Fecha de recepción: 17/05/2017

Fecha de aceptación: 23/11/2017
Correspondencia: jesusfgavira@us.es

(J. Fernández Gavira)

\section{Introducción}

La propuesta que se plantea, está basada en la Pedagogía de la Aventura, que según Parra (2001) y Parra, Caballero, y Domínguez (2009) es una metodología basada en los principios teóricos del aprendizaje experiencial, que consiste en educar mediante experiencias directas a través de actividades físicas en el medio natural (y/o mediante actividades de reto/aventura), en la naturaleza o 
en entornos urbanos, con la finalidad de contribuir al proceso de humanización de las personas (tanto del alumno como del educador), sirviendo esta como enfoque metodológico para trabajar desde el aula educativa nuevos espacios y actividades que favorezcan el aprendizaje significativo del alumno.

Los juegos de rol pueden ser definidos como aquellas actividades lúdicas en que los participantes interpretan modelos de comportamiento que no son los suyos (Brell, 2006). El juego ocupa un lugar importante en la niñez, quizás por ello los docentes lo utilizan para guiar a los discentes a que descubran la realidad, tanto física como social que les rodea. El juego sitúa al alumnado en el centro del aprendizaje, experimentando en primera persona todas aquellas situaciones y enseñanzas que se deseen estudiar. Ayuda a adquirir aprendizajes significativos, que son interiorizados más fácilmente y que tienen una mayor durabilidad en el tiempo que otros conocimientos memorizados o descontextualizados (Magee, 2006).

En la actualidad, a todos estos juegos usados para el aprendizaje y que se basan en situaciones de la vida real se les conoce también como "serious games" (Michael y Chen, 2005). De manera específica nosotros utilizaremos el juego de rol, en el cual según Escapa, González y Lucas (2015, p. 2 ), "el jugador interpreta un rol que le ha sido asignado y cuyas características o pautas de comportamiento le han sido transmitidas".

Hay unas instrucciones claras de funcionamiento y unas estrategias bien definidas a seguir, en mayor o menor medida según el juego, entre las que el jugador deberá escoger.

El final es cerrado (un único final preestablecido para cada jugador) o parcialmente cerrado (varios posibles finales preestablecidos que dependen de las decisiones durante el juego).

Por último, en los juegos de rol, hay que mencionar el desarrollo de un tipo de técnicas englobadas en lo que se denomina gamificación, del inglés "gamification", que buscan incorporar el elemento lúdico de los juegos a situaciones de aprendizaje, con el fin de favorecer la motivación que los juegos generan, pero sin constituir un juego en sí mismo y que se encuentran presentes a lo largo de nuestra propuesta (Deterning, Dixon, Khaled, y Nacke, 2011).

Por su parte el término "habilidades motrices básicas" (HMB) está ampliamente difundido en el mundo de la educación física y del deporte. Batalla (2000, p. 14) las define como "aquellas familias de habilidades amplias, generales, comunes a muchos individuos (por tanto, no propias de una determinada cultura) y que sirven de fundamento para el aprendizaje posterior de nuevas habilidades más complejas, especializadas y propias de un entorno cultural concreto". De manera general y en el contexto de España, las habilidades básicas genéricas son: desplazamientos, saltos, giros, lanzamientos y recepciones.

En el contexto de esta propuesta adquiere gran relevancia el contenido de la orientación, más concretamente el saber orientarse con un mapa, conocer las partes del mismo, etc. La orientación, es definida por Morales y Guzmán (2000, p. 444) como la "realización de un recorrido por diferentes lugares, de una zona delimitada, conocidos o no, dibujado en un mapa, pasando por unos controles fijados en el terreno en un orden establecido". La orientación es la acción de ubicar. Bajo el término de orientación designamos la acción de saber hacia dónde nos dirigimos, conociendo el lugar donde nos encontramos, por eso decimos que estamos orientados cuando conocemos nuestra posición en el terreno y sabemos identificarla en el plano. Parafraseando a García (2013), "Los alumnos se encuentran en pequeñas situaciones problemáticas que deben saber resolver aplicando su saber espacial. En este sentido son muy útiles contextos de juego: carreras de orientación, gimkanas, juego de simulación, etc.".

\section{Destinatarios y objetivos}

Los destinatarios de este gran juego serán los alumnos de primaria de quinto o sexto curso, todas las líneas o un solo grupo, según se decida en el centro, receptores de los contenidos de "El cuerpo y sus Habilidades Perceptivas Motrices" o análogo en función de la Comunidad Autónoma donde se realice esta actividad. El objetivo que se plantea es aprender de manera lúdica las HMB a partir del juego de rol "Las Aldeas de la Historia". A su vez se tratarán varios objetivos secundarios 
como son: dar respuesta a propuestas reto/aventura y sensaciones más complejas, en las que el entorno sea cambiante y ofrezca un mayor grado de incertidumbre, fomentar la interacción entre los alumnos, cooperar con los compañeros del grupo para resolver los diferentes retos o situaciones que se plantean así como colaborar en la organización de un juego de rol como medio para desarrollar una propuesta de intervención interdisciplinar y utilizar desde otras áreas del ciclo el juego de rol para impartir sus propios contenidos siendo una de las Competencias Claves utilizadas la de Aprender a Aprender al aportar el juego de rol distintas estrategias posibles para afrontar la tarea.

\section{Diseño y desarrollo de la propuesta}

Hay que mencionar que previamente al gran juego tendrán lugar una sesión preparatoria, en la que se motivará y sensibilizará hacia la temática en cuestión; esto es, unos días antes de la celebración del gran juego de rol, a los estudiantes de Educación Física se les pone en antecedente de lo que van a vivenciar y el rol que van a desempeñar de cara a suscitar en ellos un especial interés por participar activamente de la experiencia (Fernández-Gavira, Álvarez-Domínguez, Grimaldi-Puyana, y LlopisGarrido, 2015).

En la primera sesión se trabajarán las partes del plano (mapa), se conocerán el significado de los colores y la leyenda de los mismos, así como los símbolos básicos que se utilizan en los mismos.

En la segunda sesión destinada a la tarea de orientación con mapa se hará una búsqueda del tesoro dentro del centro, para que empiecen a utilizar el plano: saber orientarlo correctamente e interpretar lo que ven.

Paralelamente en otras áreas del currículum de primaria como educación artística, se dedicará una sesión a la búsqueda de información sobre como confeccionar disfraces con materiales reciclados como botellas, papel de aluminio, brick, ropa vieja, etc. Desde las matemáticas, trabajaran como eran los números de dichas culturas, como era su forma de calcular, sus medidas, etc. en el área de lenguaje trataran las palabras que han aportado a nuestro vocabulario y su escritura. En cuanto a las áreas de sociales y naturales, estudiarán las distintas culturas, su forma de vida, si siguen existiendo o no, etc. y por último en el área de música escucharan y apreciaran los diferentes tipos de músicas en estas culturas.

El juego de rol será la tercera sesión de la propuesta educativa, basado en la Pedagogía de la Aventura como incentivo para crear una propuesta lúdica interdisciplinar y educativa que aun desarrollándose desde la asignatura de Educación Física implicará necesariamente a otras materias, para un grupo de 50 personas de primero o segundo de primaria que se realizaría en horario lectivo de educación física y/o artística y que se estructura alrededor de tres sesiones.

El juego de rol se realizará en el horario de la asignatura de educación física y/o artística, en todo caso de precisa de al menos 100 minutos para poder llevarla a cabo. Para realizarla se saldrá con los alumnos a un parque del entorno cercano en el que esté el centro escolar que cumpla las características de ser lo suficientemente grande para que un grupo de 50 personas pueda correr y esconderse y que preferentemente esté vallado para facilitar la seguridad, control y referencias del grupo. Previamente los alumnos se habrán dividido en 8 o 10 grupos de 4 o 5 componentes, acompañados en todo momento por un lazarillo o personas responsable que puede ser un padre/madre o alumnos mentores de cursos superiores, y cada grupo será aldeano de una aldea: galos, romanos, griegos, egipcios y árabes. Cada componente del grupo deberá estar ataviado con algún objeto que caracterice a su grupo, sombreros, vestimenta, objetos que se habrán preparado en la clase de educación artística.

En dicho parque se distribuirán una serie de balizas, en las que habrá que realizar diferentes pruebas, en estas localizaciones siempre habrá una persona de referencia de la organización ya sea profesor, padre o alumno de cursos superiores. Todas ellas relacionadas con las HMB, desplazamientos, giros, saltos, lanzamientos y recepciones. En cada prueba y a modo de "juego trivial", si el grupo supera dicha prueba se le entregará un garbanzo del color que se le haya asignado previamente a dicha prueba. El primer grupo que consiga todos los garbanzos de los diferentes colores 
ganará la prueba. Aunque esto no será tan sencillo, ya que habrá varias personas con poderes que serán "dioses", algunos serán buenos y ayudarán a los alumnos a conseguir los garbanzos, pero habrá otros que serán dioses malos, que su cometido será proponer una nueva prueba a los grupos que se encuentre. Si dicho grupo no supera la prueba este dios les quitará el garbanzo de color que estime oportuno. Esto hará el juego más divertido y garantizará un poco de igualdad entre grupos.

Presentamos a continuación las actividades propuestas en la Ginkana:

Actividad 1 "Cuidado con los cocodrilos": esta actividad consistirá en cruzar de una zona señalizada a otra del espacio. En el punto de salida se les dejará varios ladrillos y deberán cruzar todo el grupo junto. Si alguno "cae" al agua, es decir si pisan el suelo vuelven a empezar (véase Figura 1). Esta actividad tiene un máximo de 5 minutos, en caso de no conseguir el objetivo deberán de ir a otra prueba y volver a intentarlo más tarde.

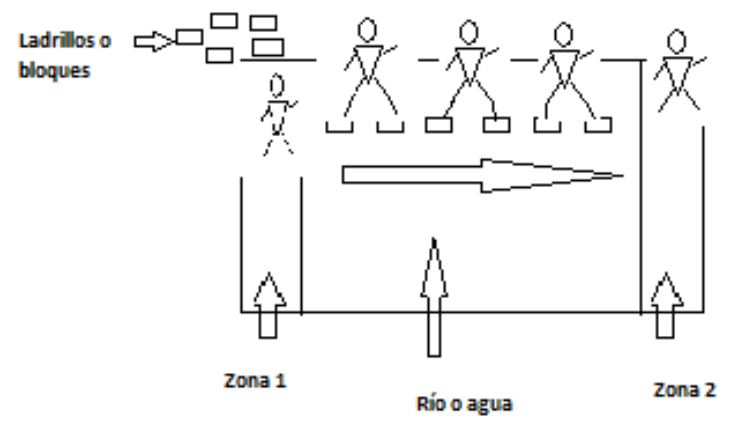

Figura 1: Cuidado con los cocodrilos. Elaboración propia.

Esta actividad se corresponde con la habilidad motriz de desplazamiento. Su premio será un garbanzo verde.

Actividad 2 "El buscapiés": el coordinador se sitúa en el centro, cogiendo una cuerda por uno de sus extremos y la hace girar a ras de suelo. Los alumnos irán saltando en dirección opuesta al de la cuerda. Si la cuerda toca el pie de alguno quedará eliminado. Deberá aguantar al menos uno para conseguir el objetivo (véase Figura 2). Habrá varias pausas para que tomen aire y recuperen. Esta actividad tiene un máximo de 5 minutos, en caso de no conseguir el objetivo deberán de ir a otra prueba y volver a intentarlo más tarde. Esta actividad se corresponde con la habilidad motriz de salto. Su premio será un garbanzo rojo.

\section{Actividad 3 "Atrapa la} bomba": el coordinador se sitúa en una parte de una pista con una red en medio y el grupo en la otra. El coordinador lanzará pelotas de espuma a los alumnos y estos deberán de cogerlas sin que se caigan al suelo. Lo alumnos deberán coger más de 2 pelotas (véase Figura 3 ). En el caso de no conseguirlo volverán más tarde. El tiempo de esta actividad será de 7 minutos. Esta actividad se corresponde con la habilidad motriz de recepciones. Su premio será un garbanzo naranja.

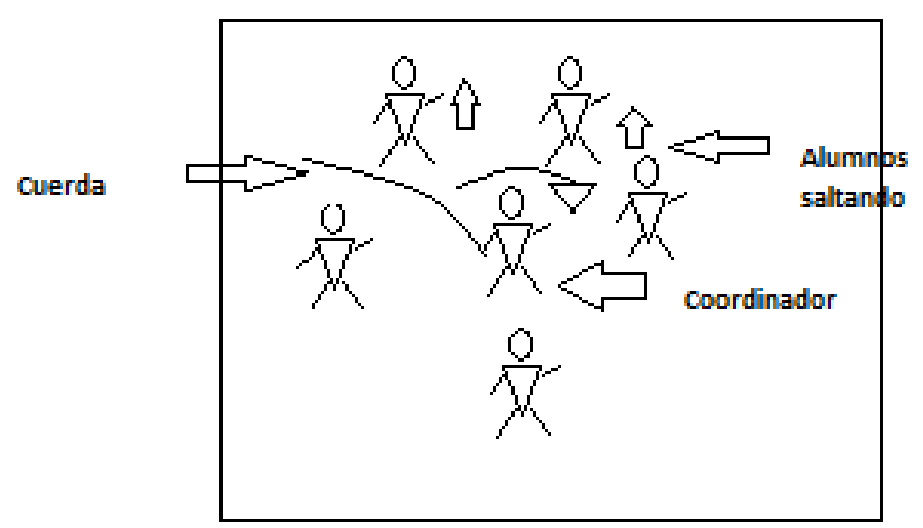

Figura 2: El buscapiés. Elaboración propia.

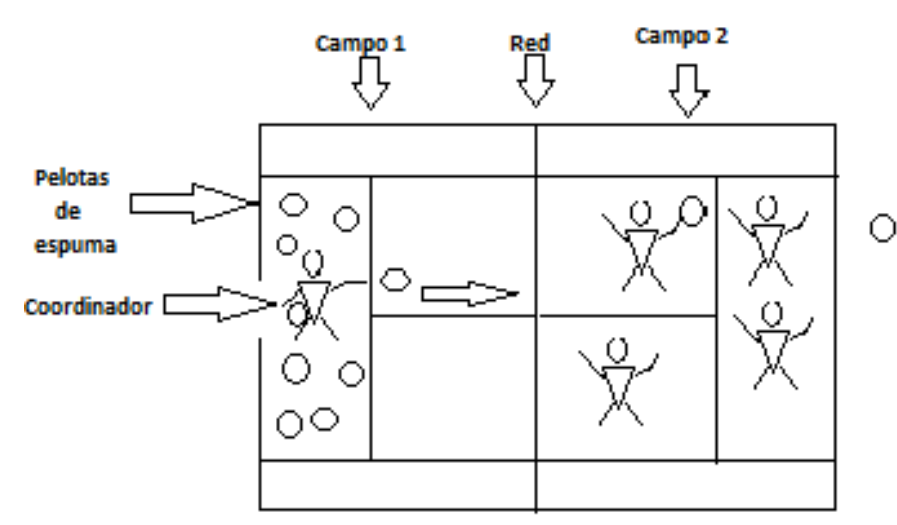

Figura 3: Atrapa la bomba. Elaboración propia. 
Actividad 4 "El transporte de tronquitos": Todos menos uno se ponen en el suelo tumbados boca-abajo y bien pegados (ellos serán la cadena transportadora). El que queda, el tronquito, se coloca encima de la cadena transportadora, de tal modo que sean estos niños, los que mediante giros (tipo croqueta), hagan avanzar al tronquito hasta el final, donde deberán de poner una pelota de tenis que llevará el tronquito en la mano. Cuando el tronquito llegue hasta el final, se pone a formar parte de la cadena transportadora y otro niño hace el rol de tronquito. Deberán de transportar 3 pelotas de tenis, sin que toque el suelo o se les caiga (véase Figura 4). Esta actividad durará 7 minutos. Esta actividad se corresponde con la habilidad motriz de giros. Su premio será un garbanzo negro.

Actividad 5 "Carrera combinada": en esta actividad los alumnos deberán de transportar varios balones de diferentes tamaños, de formas diferentes y con diferentes partes del cuerpo. Debe participar todo el grupo. Deberán transportarlo de un lugar a otro previamente señalado (véase Figura 5). En caso de que se caiga el balón o de que la toquen con otra parte que no sea la elegida empezarán de nuevo. Para ganar el garbanzo de color deberá de pensar en cómo transportar los diferentes balones, cada uno de una manera diferente. Esta actividad tiene un máximo de 5 minutos, en caso de no conseguir el objetivo deberán de ir a otra prueba y volver a intentarlo más tarde. Por ejemplo una pelota de tenis, todos con un dedo. Esta actividad se corresponde con la habilidad motriz de desplazamiento. Su premio será un garbanzo azul.

Actividad 6 "Como canguros": en esta prueba los alumnos deberán ir saltando dentro de un saco hasta un sitio previamente marcado allí habrá un papel que deberán de firmar, y volver a donde salieron para darle el saco a su compañero (véase Figura 6). Para obtener el premio deberán haber firmado todos. En caso de que alguno se caiga o ponga la mano en el suelo, deberá empezar de nuevo. Esta actividad durará como máximo 5 minutos. Esta actividad se corresponde con la habilidad motriz de salto. Su premio será un

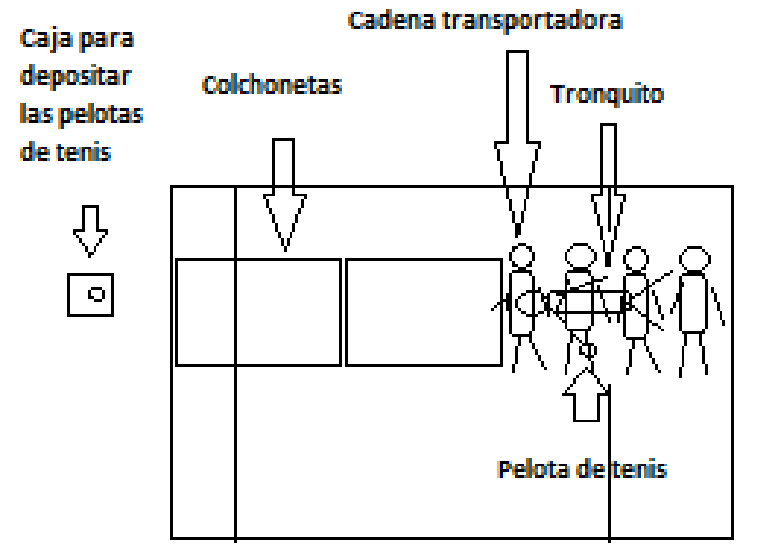

Figura 4: El transporte de tronquitos. Elaboración propia. 
garbanzo amarillo.

Actividad 7 "Imítame": en esta actividad los alumnos disponen de colchonetas diferentes, deberán elegir el tipo de colchoneta y el tipo de giro/rotación. Deberán cruzar dos colchonetas con el giro elegido. Tienen que ser dos tipos de giros diferentes, y todo el grupo debe ser capaz de repetirlo (véase Figura 7). Esta actividad durará un máximo de 7 minutos. Esta actividad se corresponde con la habilidad motriz de giros. Su premio será un garbanzo marrón.

Actividad 8 "Catapultas": en esta prueba los alumnos se encuentran en una pista con canastas de fabricación artesanal o reales dentro del parque. Los alumnos lanzando a canasta desde diferentes posiciones irán sumando puntos según desde donde encesten. Tiene que conseguir 15 puntos entre todos. Tienes 3 tiradas por turnos desde la posición que quieran (véase Figura 8). La actividad durará un máximo de 10 minutos. Esta actividad se corresponde con la habilidad motriz de lanzamientos. Su premio será un garbanzo morado.

Actividad 9 "los meteoritos": en esta actividad los alumnos estarán sentados en círculo y el coordinador estará en el centro con dos pelotas, este lanzará las dos pelotas al aire. Los alumnos deberán de levantarse y coger las pelotas sin que toque el suelo. Esta acción se repetirá 3 veces, los alumnos deberán recepcionar un mínimo 3 pelotas entre las 3 repeticiones, sin que se les caiga al suelo para conseguir el objetivo (véase Figura 9). La actividad durará 5 minutos. Esta actividad se corresponde con la habilidad motriz de recepciones. Su premio será un garbanzo blanco.



Figura 7: Imítame. Elaboración propia.

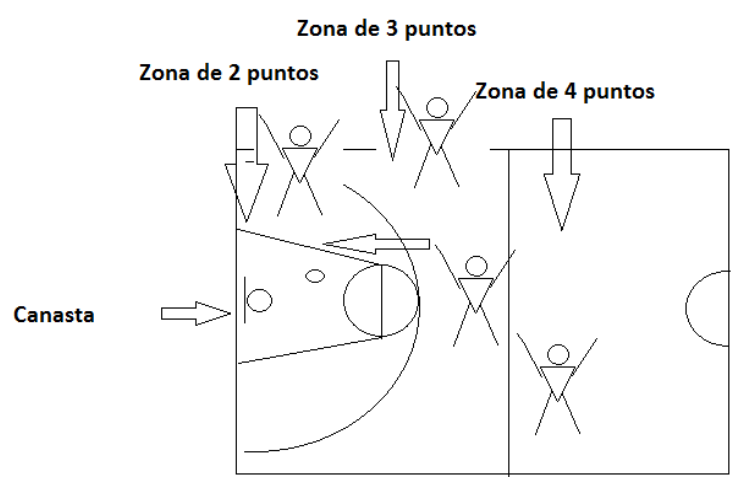

Figura 8: Catapultas. Elaboración propia.

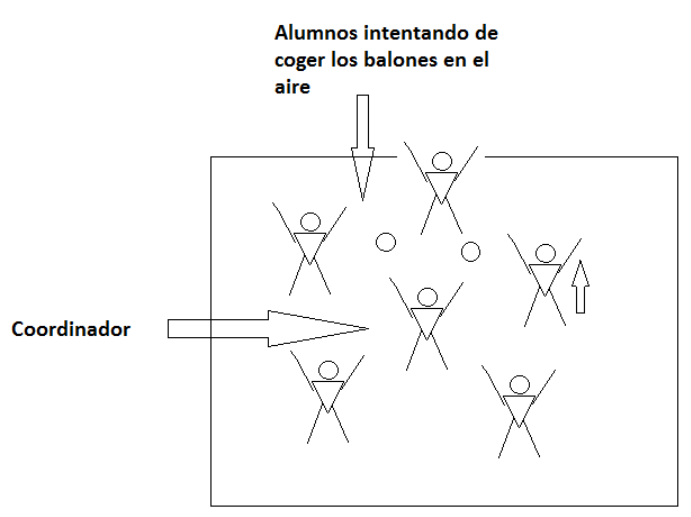

Figura 9: Los meteoritos. Elaboración propia.

Actividad 10 "Acaba con todos": esta prueba consistirá en derribar diferentes conos dispuestos en varias posiciones diferentes. Deberán ser derribados con balones, estos se pondrán en el centro desde donde lanzan. Los alumnos deberán de elegir el balón con el que tiran, para acertar bien al cono. Tienen 2 lanzamientos por alumnos, por turnos (véase Figura 10). Por ejemplo un cono pequeño con un balón de pilates, para tener más posibilidad de tirarlo. La actividad durará 7 minutos, y 
deberán de derribar mínimo 12 conos. Esta actividad se corresponde con la habilidad motriz de lanzamientos. Su premio será un garbanzo rosa.

Una vez preparadas todas las pruebas, se esperará a todo el/los grupos, según se haya decidido en el centro y de los recursos humanos disponibles, en el lugar citado, que será una de las entradas del parque, hasta donde vendrán acompañados de algunos docentes del centro entre los que estarán los de educación física y artística, así como cualquier otro docente de apoyo que se quiera sumar a la iniciativa incluso padres interesados en participar en la organización de la

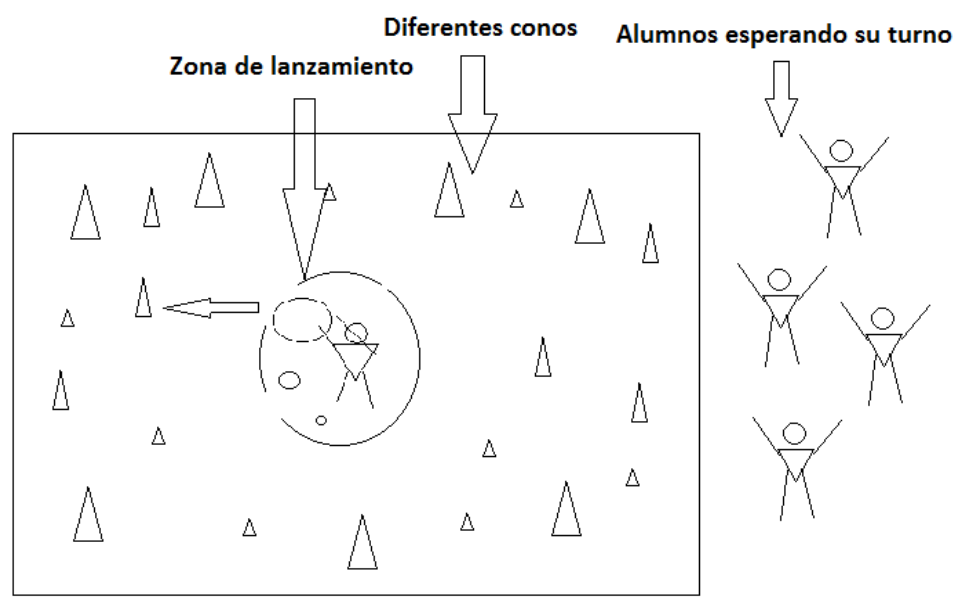
actividad. Una vez allí se les explicará de nuevo en qué consiste el juego y las normas, y se les hará entrega de un plano o mapa del parque con las balizas (Ver figura 11, se trata de un plano tipo del espacio original donde se ha previsto la actividad, no obstante esta podrá realizarse en cualquier otro espacio). En cada posta habrá un papel que les explique la actividad y a su vez habrá un responsable de baliza que en caso de que no entiendan las actividades se las explicará. Este responsable será el que haga de juez y decida si lo han conseguido o no, y a su vez pase a entregarles el garbanzo del color de dicha prueba. En todo caso para un grupo de 50 personas se precisará de seis personas responsables.

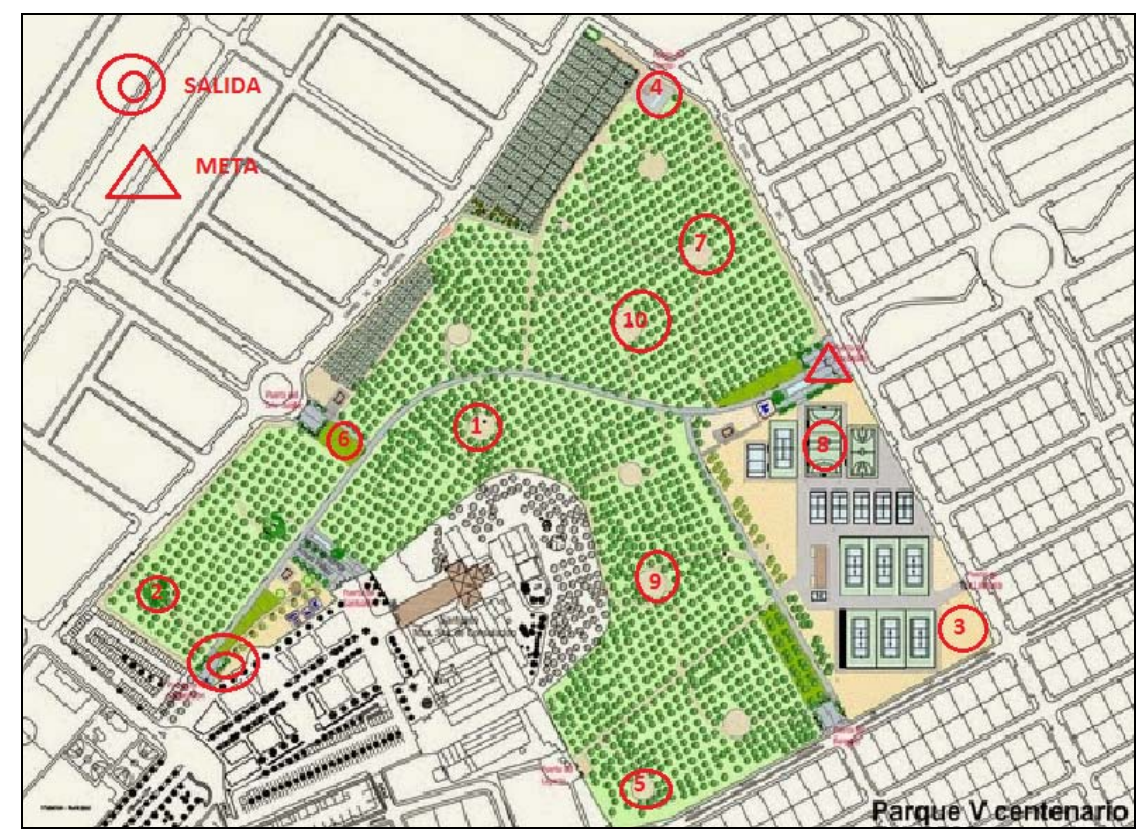

Figura 11: Plano de la prueba. Elaboración propia.

En el punto de encuentro se le dará una hoja de control (ver figura 12) para picar en las balizas, en esta hoja deberán poner el nombre del grupo y de sus componentes. Dicha hoja deberá ser entregada en la meta junto con los garbanzos de colores para verificar si lo han conseguido. Para transportar los garbanzos conseguidos a modo de premios cada grupo deberá llevar una bolsa, que será transportada por todos los miembros durante el juego, es decir de una posta a otra por un alumno diferente, con lo que el portador de dicha bolsa será el responsable en ese momento. 


\begin{tabular}{|c|c|c|c|}
\hline \multicolumn{4}{|l|}{ Nombre del grupo: } \\
\hline \multirow[t]{5}{*}{ Nombres de los componentes del grupo } & \multicolumn{3}{|c|}{ Balizas picadas } \\
\hline & 1. & 2. & 3. \\
\hline & 4. & 5. & 6. \\
\hline & 7. & 8. & 9 . \\
\hline & 10 & & \\
\hline
\end{tabular}

Figura 12: Hoja de control de grupo

Mínimo un componente o dos deberán de llevar reloj para volver a la hora que se les ha citado en el lugar señalado en el mapa.

Una vez se haya explicado en qué consiste el juego, normativa y aclarado dudas, se les dará un plano por cada grupo. En este plano estarán señalizadas las balizas donde se encuentran las pruebas, la salida y la meta, donde se encontrarán todos al finalizar el juego. Cada grupo es libre de elegir el orden para ir a la posta o baliza que quiera.

La Yincana acabará con un gran juego final titulado pelea de súper-héroes, para lo cual a cada grupo se le dará varios periódicos viejos, cinta adhesiva y tijeras con los que habrán de caracterizar a una persona del mismo que actuará como súper héroe del mismo, a la voz de un árbitro de juego, todos los superhéroes de los grupos entrarán en un ring delimitado por una cuerda con la particularidad de que serán superhéroes ciegos a los que se les pondrá unas gafas totalmente opacas. El objetivo entonces será que a partir de las super-técnicas o super-habilidades adquiridas en las pruebas anteriores consigan quitar a los otros super-héroes sus super-disfraces de periódicos. Mientras tanto desde el exterior del gran ring el resto de los alumnos gritando darán instrucciones de combate a sus momias. El juego acabará tras diez minutos y se proclamará vencedor al super-héroe que más habilidades motrices de combate haya aplicado y al que conserve la mayor cantidad de papel adherido al cuerpo.

Tras esta actividad el gran grupo se sentará en círculo y se reflexionará sobre cada una de las actividades realizadas, las habilidades adquiridas, así como las propias sensaciones y vivencias durante la actividad.

El enfoque metodológico adoptado para este estudio ha sido el análisis cualitativo del contenido semántico de una serie de artículos seleccionados tras una revisión bibliográfica (Conde, 2010). El proceso seguido se estructura en tres fases: a) revisión bibliográfica, b) análisis de la información, c) realización de la propuesta de análisis (Hernández-Hernández y Palao, 2011).

\section{a) Revisión bibliográfica}

La revisión bibliográfica se ha realizado utilizando algunos de los buscadores más importantes a nivel nacional como CSIC (ICYT, IME, ISOC), DIALNET, entre otras. Igualmente, se han utilizado bases de datos internacionales, como ISI Web of Knowledge, Scopus y Sports Discus. Las palabras clave utilizadas en los sistemas de búsqueda de las bases de datos en español, fueron: actividades físicas en el medio natural, actividades en la naturaleza, aventura (Baena-Extremera et al., 2015).

\section{b) Análisis de la información}

Una vez concluida la búsqueda se realizó un proceso de selección de la información. Primero se eliminaron los registros duplicados; a continuación, se revisaron los campos principales (título, resumen, autoría, palabras clave, fecha de publicación, temática y tipo de documento), desechando todos los trabajos fuera de la línea del estudio. Posteriormente, se aplicaron dos criterios de selección: artículos que versen sobre epistemología y taxonomía de las AFMN/AFAN y artículos que describen 
características específicas de las AFMN/AFAN. Por último, se realizó una lectura crítica de los documentos (Guirao-Goris, Olmedo, y Ferrer, 2008), que permitió evaluar la calidad e idoneidad de los estudios, y finalizar el proceso de selección de la información.

\section{c) Realización de la propuesta de análisis}

El último procedimiento que se realizó fue analizar los diferentes artículos seleccionados, y definir una serie de categorías relativas a los factores universales comunes de las AFMN/AFAN, mediante el análisis cualitativo de los documentos (Conde, 2009) y los criterios para crear categorías propuestos por Anguera (2003).

La metodología aplicada en esta investigación plantea las siguientes limitaciones. En primer lugar, la selección de los artículos para realizar las categorías sobre los factores universales; será necesario un proceso adaptación del sistema de categorías, en una fase posterior donde testear/analizar la inclusión de los factores universales en bibliografía de AFMN y AFAN. En segundo lugar, es necesario realizar un proceso de validación del contenido por expertos, que aporte validez externa al sistema de categorías, es decir, a los factores universales sobre las AFMN/AFAN (Carretero-Dios y Pérez, 2007). Las limitaciones expuestas deberán tenerse en cuenta en la interpretación de los resultados.

\section{Resultados esperados}

El trabajo presentado, es una propuesta fundamentada en el juego de rol para aprender HMB, la cual se presenta en un formato interactivo y fácilmente repetible, por cualquier docente que quiera volver a simular la experiencia. Por medio de la misma, hemos procurado incorporar al contexto escolar, un trabajo interdisciplinar entre áreas de acuerdo con Pombo (2011), quien apunta que, se entiende por interdisciplinariedad una forma de combinación entre dos o más disciplinas, con vistas a la comprensión de un objeto a partir de la confluencia de varios puntos de vista diferentes. Implica, por tanto, una reorganización del proceso de enseñanza-aprendizaje que supone un trabajo continuado de colaboración entre los docentes involucrados.

El diseño y ejecución de este juego de rol tiene un doble interés académico, pues al tratarse de un aprendizaje vivenciado por los mismos estudiantes, no resulta difícil establecer una clara relación entre lo que se aprende a nivel teórico y lo que experimenta a nivel práctico. En este sentido, el carácter lúdico de la experiencia hace que el alumnado sea el principal demandante del conocimiento (Fernández et al., 2015).

Entendemos que fomentar una enseñanza lúdica, en este caso a través del juego, y sobre todo en otro ambiente o contexto como es en el medio natural en vez del centro, es más motivador para los alumnos. Como mencionan Giménez, Pagés y Martínez (2010), en el actual contexto docente faltan métodos pedagógicos diferentes que supongan un aliciente añadido al de las meras "clases magistrales". Tal es el caso, por ejemplo, de los juegos.

Según Wenner (2011) el juego resulta crucial para el desarrollo social, emocional y cognitivo de los niños. Permite una mejor adaptación, estimula la inteligencia y reduce el estrés. El juego resulta además crucial para que una persona llegue a ser socialmente competente, maneje el estrés y desarrolle habilidades cognitivas, como por ejemplo, la capacidad para resolver problemas.

Esta propuesta didáctica, se propone también desarrollar habilidades de aprendizaje cooperativo entre los participantes. Podemos decir que reúne los requisitos necesarios para garantizarlo, pues desde el principio y hasta el final del proceso de enseñanza-aprendizaje, los estudiantes son retados de manera continuada a trabajar grupalmente, poniendo al servicio de un grupo las particulares habilidades personales de cada uno, entre las que el liderazgo positivo es fundamental (Fernández et al. 2015). 


\section{Conclusiones}

Consideramos que esta propuesta educativa está directamente unida a la gamificación que permite que mediante estrategias lúdicas y proactivas que el alumnado pase de ser mero receptor de conocimientos a trabajar de manera activa y comprometida en la adquisición de los mismos (Fernández-Gavira, García-Fernández, Sánchez-Oliver y Grimaldi-Puyana, 2017). De esta manera los discentes implicados participarán mediante el juego de rol de la creación de su conocimiento sobre las HMB de manera lúdica y satisfactoria. En esta línea creemos que este proyecto trae otras ventajas añadidas entre las que están el que al implementar las ventajas del uso del juego como herramienta para el aprendizaje, probablemente aumentará la socialización dentro del grupo-clase, disminuirán los conflictos entre el alumnado, adquirirán una mayor responsabilidad dentro de un grupo y sentido de pertenencia al mismo, etc., como se ha comprobado en trabajos de índole similar entre los que podemos destacar el de Grande de Prado (2017) sobre videojuegos y alfabetización digital.

\section{Referencias}

Brell, M. (2006). Juegos de rol. Educación social: revista de intervención socioeducativa, (33), 104-113.

Deterding, S., Dixon, D., Khaled, R., y Nacke, L. (2011). From Game Design Elements to Gamefulness: Defining "Gamification", Proceedings of the 15th International. Academic MindTrek Conference.

Escapa, M., González, M., y Lucas, J. (2015). Aprendizaje basado en juegos de rol: el Juego de la Negociación Climática, VI Jornadas de Docencia en Economía, Bilbao.

Fernández-Gavira, J., Álvarez-Domínguez. P, Grimaldi-Puyana M., y Llopis-Garrido. A. (2015). YincaExprésate con el deporte: haciendo historia a través de una yincana nocturna. Revista de Docencia Universitaria. 13(1), 377-403. 10.4995/redu.2015.6457

Fernández-Gavira, J., García-Fernández, J., Sánchez-Oliver, A. J. y Grimaldi-Puyana, M. (2017). Gamificación, Emprendimiento y Deporte mediante las Aplicaciones Móviles. En E. López-Meneses, D. Cobos Sanchiz, A. H. Martín Padilla, L. Molina-García, y A. Jaén Martínez (Eds.). INNOVAGOGÍA 2016. III Congreso Internacional sobre Innovación Pedagógica y Praxis Educativa. Libro de Actas. 28, 29 y 30 de noviembre de 2016. AFOE Formación: Sevilla, ES.

García, N. J. (2013). El concepto de Orientación y su puesta en práctica en Educación Primaria. Escuela Universitaria de Educación, Valladolid.

Grande de Prado, M. (2017). Videojuegos y Alfabetización Digital: Mitos y Rechazo. En En E. López-Meneses, D. Cobos Sanchiz, A. H. Martín Padilla, L. Molina-García, y A. Jaén Martínez (Eds.). INNOVAGOGÍA 2016. III Congreso Internacional sobre Innovación Pedagógica y Praxis Educativa. Libro de Actas. 28, 29 y 30 de noviembre de 2016. AFOE Formación: Sevilla, ES.

Giménez, C., Pagés, C., y Martínez, J. J. (2010). Análisis, diseño y desarrollo de un juego educativo para ordenador sobre enfermedades tropicales y salud internacional: una herramienta docente más de apoyo al profesor. REDU. Revista de Docencia Universitaria, 8 (2), 131-146. Doi: 10.4995/redu.2010.6199

Magee, M. (2006). State of the field review. Simulation in education. Final Report. Alberta Online Learning Consortium Calgary AB.

Michael, D. y Chen, S. (2005). Seriuos games: Games that educate, train and inform. Muska and Lipman/Premier Trade.

Morales, A. y Guzmán, M. (2000). Diccionario temático de los deportes. Manual de términos en la actividad física y el deporte. Málaga, España: Arguval.

Parra, M. (2001). Programa de actividades físicas en la naturaleza y deportes de aventura para la formación del profesorado de segundo ciclo de secundaria. [Tesis doctoral no publicada]. Universidad de Granada, Granada, España.

Parra, M., Caballero, P., y Domínguez, G. (2009). Estrategias metodológicas para las actividades recreativas en el medio natural. En M. E. García (coord.). Dinámicas y estrategias de re-creación (pp. 199-260). Barcelona, España: Graó.

Pombo, O. (2011). Interdisciplinaridade: conceito, problemas e perspectivas. Revista do Departamento de Psicología.

Wenner, M. (2011). La importancia de jugar. Mente y Cerebro, 46. 38 - 45. 